\title{
Water Bodies Cleanliness and Waste Removal using Advanced Technologies
}

\author{
${ }^{1}$ Dr. J. Shiny Duela, ${ }^{2}$ Rahul Jain \\ ${ }^{1}$ Associate Professor, ${ }^{2}$ B.Tech Student \\ ${ }^{1,2}$ SRM Institute of Science and Technology, Ramapuram Campus, Chennai \\ Email: ${ }^{1}$ shinyduj@,srmist.edu.in, ${ }^{2} \underline{\text { ra1688@srmist.edu.in }}$
}

\begin{abstract}
Water pollution is an indispensable majorenvironmental issue faced by 7.5 billion population of people collectively on Earth today. The major contributors are the plastic waste generated gigantically is almost ubiquitous in oceans, rivers and sea beds. The largest source of waste water pollution is the untreated sewage others include agricultural run-off and waste generated via the small and big industries across water bodies. Fresh water on earth is only $2.5 \%$ of the totalwater when $70 \%$ of the earth's surface is covered by water. Around $70 \%$ of industrial waste is dumped to water. $80 \%$ of the water pollution is caused due to domestic sewage. The traditional methods of cleaning rivers manually are just not working enough in direction of even impacting the lives in positive way for humans. In order to replace the traditional manual cleaning rivers and lake for treating garbage and then improve the clean-up efficiency, while addressing the labor force of large salvage, low security issues, a new U shaped hard pipeline structure with concepts of IOT is developed having a strong initiator base upfront to hold up the machine to brook tempestuous flow of current and wind foe ocean water so that the machine can be easily susceptible and be facile for adaption of river wind stream. The solar cell panel above pipeline to be used to detect the presence of waste in the water bodies via IR sensors and the machine will drag the waste collectively via the net present under the pipes to the sea shore where manual force can dispose the waste materials with buttress of(autonomous service vehicles) ASV's. The model proposed can also serve as a means of a launch pad for underwater robots which are being developed for various applications ranging from inspection to maintenance and cleaning of submerged surfaces and constructions. Big Data will work here as an important herald to tell the operator, the amount of waste gathered in one take and serve as the means for future analysis of the data.
\end{abstract}

Keywords: Autonomous Service Vehicle (ASV), bigdata, IR Sensors, solar cell panel, launch pad, IOT.

\section{INTRODUCTION}

On August 7, 2015 the Environmental Protection Agency accidentally released around three million gallons of acidic mine drainage into Cement Creek near Silverton, Colorado. As a tributary to the Animas River, the heavy metal-laden spill turned the Animas river a putrid, or- angecolor that made its way south through Durango, Farmington, the Navajo Nation and into its final resting place in Lake Powell. This catastrophe cliché incident resulted in a community, state, and world-wide alarm, and realization that water is a precious and vulnerable resource that can be contaminated within blink of the eye in future. This is a supportive one case example to what greater threats usually rivers and oceans are frequently treated . Eight of them are in Asia: the Yangtze; Indus; Yellow; Hai He; Ganges; Pearl; Amur; Mekong; and two in Africa - the Nile and the Niger[1]. The recent protest evoking in recent days especially the last year Greta Thunberg famous speech at UN, the support to conserve and preserve natural resources have grown more than ever before. There has been no impactful plastic removal machine or technique from ocean water in past years. The proposal, however, necessarily made to achieve more success manually than by the automatic mechanism .However, the recent developments in technology world, the digital revolution and the advent of Internet have allowed an unprecedented exchange of information ,innovative ideas [2] .The system developed contains the pipeline particularly in the U shaped field structure which allows the design to mostly withstand the strong current waves of oceans. The designed contains two upfront motors which enables simple and stable operation. The system contains the solar rotary collection system or the solar tracking system which can provide night time machine usage depending upon the charges of the battery. The structure contains 3-5 meter rope below in the level of sea which is attached via the hard net to ensure the plastic waste once gripped, is not left behind [4]. The system also serves as a medium to allow the underwater robots a launch pad for various applications ranging from inspection to maintenance and cleaning of submerged surface and constructions. These platforms should be able to travel on these surfaces. Concept of Big data is applied to instruct pipeline to develop the major suffice evidence to figure out the level of concussion of the underwater plastic waste collected bigotry irrespective of the waste generated[6]. Additionally, these platforms adapt and reconfigure for underwater environment conditions and are autonomous. Talking about the adhesion to the surface, they should produce a proper attaching force using a light-weight technique. The system will face deviations from expedite stream of 
ocean waves and juxtapose wind currents for that strong foundations of the system is essential and is indispensable. Web based collaborative Big data analysis on Big data as a service platform [3].

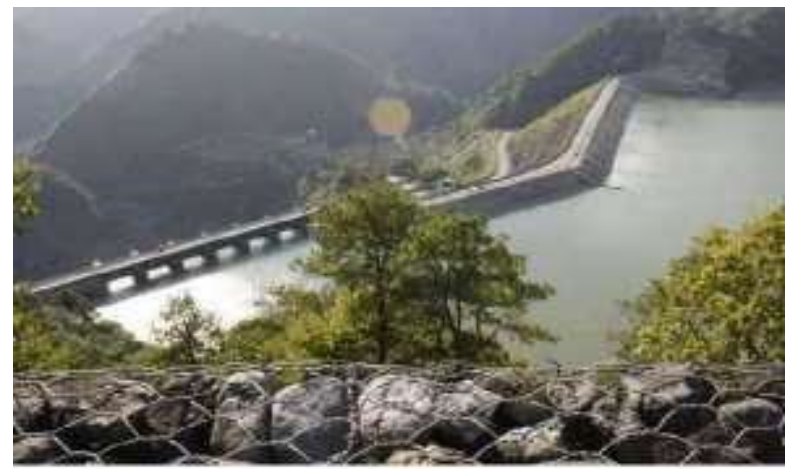

Fig. 1. Dams Side Waste Cleaning operation on Webbased IOT

The models like the one depicted above on dams' side water cleaning Web Based IOT has significantly been successful and bringing positive effect to nearby areas.

\section{FORESEEN MODEL}

The generation of waste water has gigantic impact in the working and living world .The symptoms usually is the declining fresh water percentage across the globe. The system work for the development of boat is be designed in such a manner that the waste is carried away with the pipelines broadly involved in the $U$ shaped structure. The system structure also carries an underwater rope to fall in ocean around 3 meters or more to support the dragging process of waste. The pipeline structure also carries a solar panel which is constructed at the top bolstered with IR $\mathrm{s}$ sensors to detect the plastic presence and useful for water bodies facing low movement[5]. Thus, gallons of waste especially plastic can be drawn out of the river,oceans or seas. The remote control systems is defined and applied to the system or machine for autonomous operation. Since the distance of remote control systems is limited and autonomous operation is preferred to teleoperated mode, an INS- based GPS-expedite aid navigation system was designed to enable the boat follow a predetermined trajectory[7].

The trajectory determined will serve as a medium to save further distances of same path by tracking the system and sending the system to the same predefined path saving the energy and the fuel. While the reference motion of trajectory is provided by the integrated IMU, the GPS receiver acts as the updating system. The reason behind this approach is the frequency of INS measurements which is higher than that of GPS measurements [4]. This system is used to work for a medium to release underground robots and launched it in the bodies thus save the money and further expenses of submarines sending especially for the underground cleanliness purpose and henceforth the proposed system can work multi purposely to help and make water free of all plastic waste[11,12].

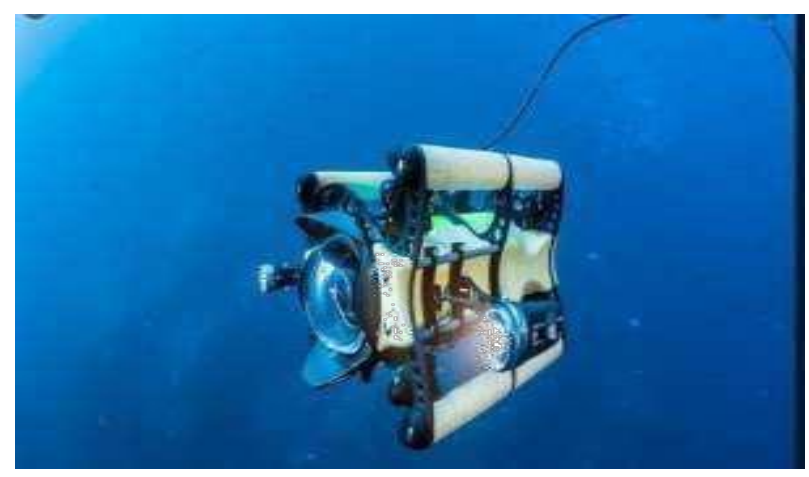

Fig 2. Representing U shaped Pipeline structurewith solar panel at the apex to clean rivers. 


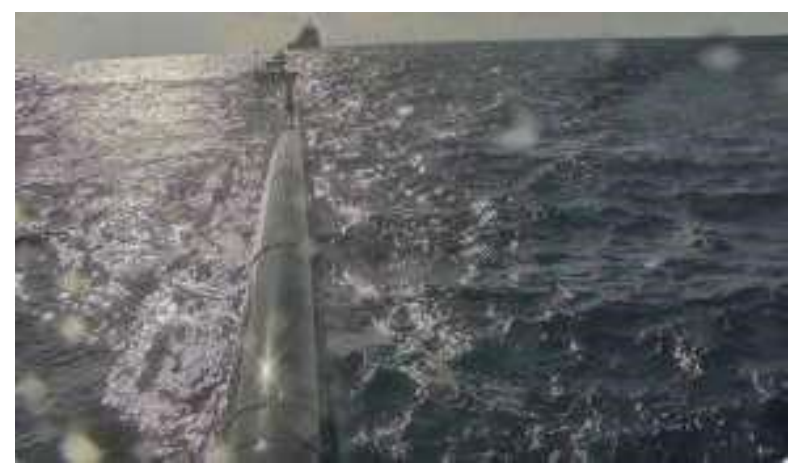

Fig 3. Underground water robots send in the ocean toperform its operations via U shaped pipeline system.

The Table1 is the recent statistic generated and collected wherein China is found to generate most plastic among all nations of the world. About 9 million mismanaged plastic waste and 3.5 million plastic marine debris is concerning.Among other nations includes Indonesia, Phillipines,Vietnam, Sri Lanka, Egypt and Malaysia. India and USA are also among the top 10 nations plastic Waste generation list. The amount of plastic waste generated is far beyond our control until the significant steps are taken. The country of Asian region is the most who tops the list and the time is crucial to ponder and use proper manageable steps. The pipeline structure can serve the upon the purpose well and the solar cell panel; increases efficiency.

Table 1. Highest plastic waste generated countries of the World and their amount ofwaste generation.

\begin{tabular}{cccc}
\hline S.NO. & $\begin{array}{c}\text { Country Name(Descending } \\
\text { Order of WastePlastic } \\
\text { Generation } \\
\text { and Pollutingrivers) }\end{array}$ & $\begin{array}{c}\text { ApproximateAmount } \\
\text { (Million)Mismanaged } \\
\text { PlasticWaste }\end{array}$ & $\begin{array}{c}\text { Approximate } \\
\text { Amount(Million) } \\
\text { Plastic Marine } \\
\text { Debris }\end{array}$ \\
\hline $\mathbf{1 .}$ & China & 8.8 & 3.54 \\
\hline $\mathbf{2 .}$ & Indonesia & 3.2 & 1.29 \\
\hline $\mathbf{3 .}$ & Philippines & 1.9 & 0.75 \\
\hline $\mathbf{4 .}$ & Vietnam & 1.8 & 0.73 \\
\hline $\mathbf{5 .}$ & Sri Lanka & 1.6 & 0.64 \\
\hline $\mathbf{6 .}$ & Egypt & 1.0 & 0.42 \\
\hline $\mathbf{7 .}$ & Malaysia & 1.0 & 0.39 \\
\hline
\end{tabular}

\section{COMPARING EXISTING MODELS}

The system or machines developed before are serving the purpose of only either cleaning a certain region and are all basically creating the" small" impacts[9,10]. The rate of generation of plastic waste is more than the rate ofremoval. Clearly, indicating the ever soslightly change the machines are having. The proposed U-tube shaped pipelinesstructure can serve the purpose of notjust only carrying gallons of water awayto the shore but also sendingunderground robots to the water bodiescauses double bonanza[15][16]. Also, thepresence of IR sensors, solar panelplaced handsomely on the top of thepipeline making the strong chances ofsupport of modal success. The systemalso has the facility of autonomoussurface vehicles (ASVs) equipped withwater quality sensors and the real-timewireless communication making it highlyeconomical and effective. Solution tomonitor ultimately protect the river waterand restore the quality of water backinvolves following these circumscribedconditions[13,14]:

- Efficient: Carrying waste away from the oceans, rivers and sending underground robots below in the sea level enables efficiency.

- Multipurpose: Solve many problems together as a machine.

- Economical: Construction of one U tube pipeline structure may be expensive but considering the tasks it performs together plus on comparing the machines before cost of construction, worth it, hence economical.

- Save time and efforts: Multipurpose system structure is always beneficial as its saves time and efforts by shortening the follow of iterative steps and marshalling together to one.

- Automatic Operations: Requiring a smaller number of manual operations

- Sensing Subsystem: The sensingsubsystem involves the measurement of plastic waste gathered together by the pipeline system and send the message to the end user, controller, who implicitly figure out the level of 
plastic waste collected and then send the data forward for future references. The digital revolution and advent of Internet have allowed an unprecedented exchange of ideas, data and information. This information can be used to serve a purpose of amity and refining statistic for future level references. This IOT based dam waste water treatment is also installed at new dams in few countries recently and the development and gumption use of the system is showing positive signs.

\section{RESULT ANALYSIS}

The earlier models developed to conduct cleaning operations in the rivers were considered to serve the purpose of cleaning the river water around 1-2 tonnes in one take. However, due to tides and external factors like the Sun ray's pre-requisite for solar cell panel cleaning system, the existing system is not worthwhile for holding those wastes together and let alone carrying into the shore side. It requires the large help of manual labour for operations. Comparatively, the results shown by the proposed system have significantly high numbers to its success and implementation.

$$
\text { K pipe }=F L / D
$$

\section{Average $=$ Sum of all observation of (existing or proposed) model /Total number of observations of (existing and proposed model)

The above formula is used to calculate average of waste collected in one take and helps to determine efficiency of model. Histograms are obtained in one graph to show their graphical patterns. The frictional coefficient $\mathrm{F}$ faced by the pipeline can be found using a modified version of Colebrook White equation:

$$
F=0.25 /\left[\log \left\{k / 3.7 * D+5.74 / R e^{\wedge} 0.9\right\}\right]^{\wedge} 2
$$

Where Re=Reynold's number,$k=$ Roughness Factor $(m)$

$$
\text { Loss coefficient }=K \text { fittings }+K \text { pipe }
$$

where $\mathrm{F}=$ Frictional Coefficient $\mathrm{L}=$ Pipe Length $(\mathrm{m}) \mathrm{D}=$ Pipe Diameter $(\mathrm{m})$

Efficiency = Average value of (proposed / existing model) / Total million tonnes in water bodies available

On applying the new trends and technological measures to conserve the oceans and river water the trajectory of plastic waste can be controlled. The data is to be regularly monitored and via Big Data Applications and thus by targeting hub spot of plastic wastes across the globe and treating it helps in reduction of waste. It aims in collecting the waste amount of $20-25$ tonnes together and palpable in its style of construction. Consider the example of population of country ranging from 4 to 12 million varying from 1975 to 2014 and the significant changes made to impact the waste water collection, detection and water quality trend assessment.

Table 2. Million (tonnes) waste collected in one takeand its comparison with existing and proposed system.

\begin{tabular}{cccc}
\hline S.NO. & $\begin{array}{c}\text { (Million) } \\
\text { tonnes waste } \\
\text { collected } \\
\text { Efficiency in } \\
\text { onetake } \\
\text { (Y-axis) }\end{array}$ & $\begin{array}{c}\text { Existing } \\
\text { System } \\
\text { (X-axis) }\end{array}$ & $\begin{array}{c}\text { Proposed } \\
\text { System } \\
\text { (X-axis) }\end{array}$ \\
\hline 1. & 20 & 1 & 4 \\
\hline 2. & 40 & 2.4 & 4.4 \\
\hline 3. & 60 & 2.6 & 6.0 \\
\hline
\end{tabular}


4.

5.
80

100
4

4.2
7.4

8

On applying the new trends and technological measures to conserve the oceans and river water the trajectory of plastic waste can be controlled. The data is to be regularly monitored and via Big Data Applications and thus by targeting hub spot of plastic wastes across the globe and treating it helps in reduction of waste.

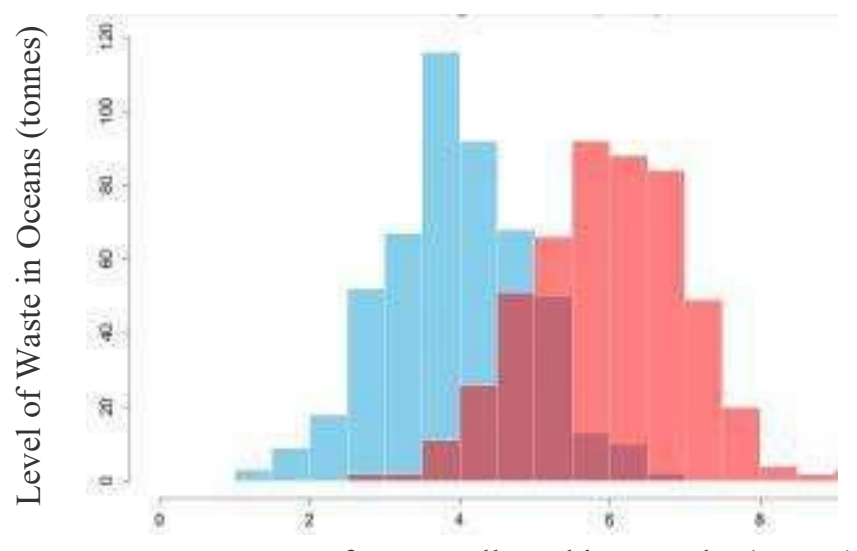

average amount of waste collected in one take (tonnes)

Fig 5. Efficiency Comparison of Existing System VsProposed System showcased by two histograms and their impact on level of Waste Collection in one take

In fig 5. X -axis represents average amount of waste collected in one take (tonnes) andY- axis represents Level of Waste in Oceans (tonnes). The average amount of waste collected with existing models lies in the range of 0.5-4.5 tonnes (maximum) while the initial carrying waste through new system is 2.5 tonnes(minimum) considering all external factors like tides, currents and storms. Repudiating the old methods can be an efficient means to look for proper application of new system.

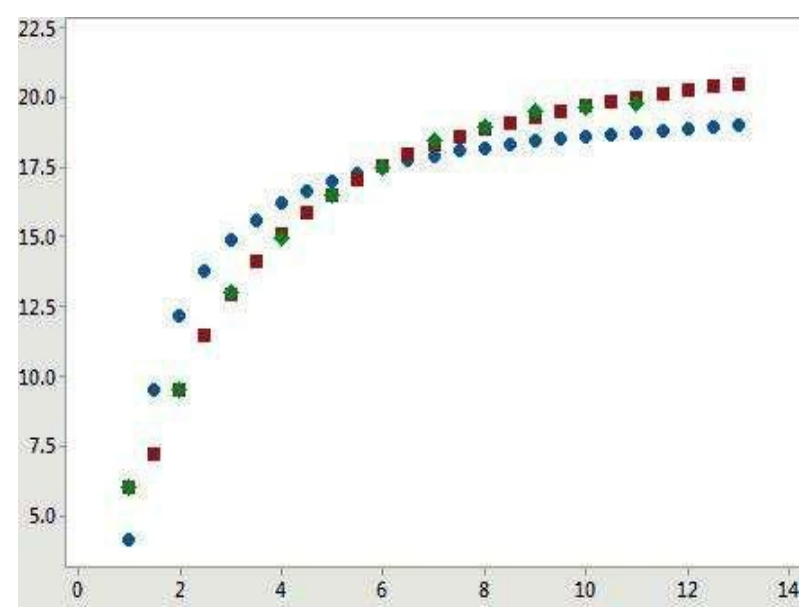

Fig 6. Existing Model Vs Proposed Model CurveFitting (Regression) Projection and Comparison

The datareceivedby collectionofplasticwastecan beusedfor analysis purpose using $\mathrm{R}$ programming language. The Fig6represent linear andnon-linearregressionof both modelsdescribing efficiencycomparison. The blue linedepicts existing systemefficiency while the redblock/dotdepictstheproposedsystemefficiency and range.Thegreendotted curve shows thecommonrangesofbothsystem indifferent conditions. Asthe parameter onX 
axisincreases the efficiency ofproposedsystem on Y-axis rises.Thus,collection of the wasteisbetter in the proposed system.

\section{CONCLUSION}

This work is mainly focused on modeling, design and control of operated boat U-shaped pipeline structure with emphasis laid on lightweight, portable appliances and multipurpose usages devices. An innovative method of minimizing manual stress and thus reliably stabilizing the garbage collect in the boat $U$ shaped structure. The project carried out by us made an impressing task in the environmental purpose domain by removing tons of gallons of waste from the rivers and if such machines are installed and are in large number most polluted rivers in several years of hard work can be plastic waste and domestic waste free. The waste generated can be sabotaged and carried to waste plant for further treatment. The system developed can only serve as a means to help the world to be a better place to live in. Creations like these may travail in pain but cannot escape its destiny. The Big data and IOT concepts collaborated in this proposed model can serve as revolutionary system in cleaning tonnes of water.

\section{References:}

1.Gaganjot Kang ,Jerry Zeyu Gao,2017,“DataDriven Water Quality Analysis and Prediction”,IEEE Explore,2017

2.Eisha Akanksha ,2019 "Modeling of SmartWater Control Mechanism using IoT" International Journal of Innovative Technology and Exploring Engineering (IJITEE) Volume-9 Issue-2,pp: 149-154

3.Zhang Feng, Xue Hui-Feng, Xu Dong-Sheng, Zhang Yong-Heng, You Fei,2013 "Big Data Cleaning Algorithms in Cloud Computing", Volume 9, Issue 3,pp:77-81

4. Prof. Sandesh M Prabhu, Mohammad IrshadK,2019 "Design and Fabrication of River Cleaning Machine” ,International Journal ofModern Trends in Engineering and Research,pp:76-80

5.Shrutika Pawar1, Sunita Shinde,2019“Remote Operated Floating RiverCleaningMachine”,Vol.6,Iss. 4, pp:3344-3347

6.Akshat Kumar, Jitendra Kurmi ,2018

"loTbased Water Surface Vehicle"

7. Salman Salloum, Joshua Zhexue Huang, 2019“Exploring and cleaning big data with random sample data blocks', Journal of BigData,Springer

8.Y Zhong ,Liang Zhang ,ShaojingXing,2014,”The big data processing Algorithm for Water Environment Monitoring of the threeGorges Reservoir Area", HindawiPublishing

Corporation, Vol.2014

9.Juan Manuel Ponce Romero, Stephen H.Hallett,2017,'Leveraging Big Data Toolsand Technologies : Addressing the Challenges of theWater Quality Sector ",Sustainability 2017, 9, 2160; doi: $10.3390 / \mathrm{su} 9122160$

10. YLing, 2019,” Big Data Analysis ofWater Quality of Secondary Water Supply

“,Procedia Computer Science, Elsevier,vol. 154,pp: 744-749

11. K Sinha ,Roheet Bhatnagar,2018,'WaterQuality Management through Data Driven Intelligence System in Barmer Region ,Rajasthan ”, Procedia Computer Science,Volume 132, pp: 314-322

12. ZhongrunXiang, JunYan,2018,” Big Dataand Machine Learning in water Sciences : Recent

Progress and their use in Advance Science",Journal at Advance Earth and Space Science, updated in January 2020

13. S, Adamala. (2017) "An Overview of Big Data Applications in Water Resources Engineering” Machine Learning Research, vol.2, No.1 ,pp 10-18

14. Geetha, S., Gouthami, S. (2016) "Internet of things enabled real time water quality monitoring system. Smart Water-Springer,vol.2,No.1https://doi.org/10.1186/s40713-017-0005-y 
15.K. Vijayakumar, Chokkalingam Arun, "Integrated cloud-based risk assessment model for continuous integration”,Int. J. Reasoning-based Intelligent Systems", Vol. 10, Nos. 3/4, 2018.

16. K. Vijayakumar, S. Suchitra and P. Swathi Shri, "A secured cloud storage auditing with empirical outsourcing of key updates”, Int. J. Reasoning-based Intelligent Systems, Vol. 11, No. 2, 2019 